\title{
Automatic detection of the autocorrelation-type measurement error component
}

\section{K. M. Hangos,}

Computer and Automation Institute, Hungarian Academy of Sciences, H-1502 Budapest PO Box 63, Hungary

\section{J. L. Nagy and L. Leisztner}

Institute of Forensic Science, H-1903 Budapest PO Box 314/4, Hungary

\section{Introduction}

Automatic detection of measurement errors is extremely important in automatic analysis of large analytical sample sequences. Error detection usually involves samples from known control solutions which are regularly introduced into a sequence of unknown samples. The control can then be performed using proven graphical methods [1] based on the sequence of the measured control values during a longer period (day or shift). Numerical methods of detection are becoming increasingly important in microcomputer-based laboratory monitoring systems.

This paper discusses one component of the measurement error: the so-called 'autocorrelation-type' error. This error component appears when the measured values of subsequent samples influence each other; it is a frequent error in analytical measurements. Its main sources are either sorption phenomena or inertia effects in the measurement or registration devices.

The main purpose of this paper is to propose an efficient numerical method for the detection of autocorrelationtype measurement error components.

First, several models of the measurement error in automatic analysis are investigated. Based on this, the numerical method proposed is decribed. Finally, the results of the validation of the proposed method and its comparison with the graphical LAG-1 method is presented for control sample sequences.

\section{Materials and experimental methods}

The sample sequences investigated were control samples with $0.5,1.0$ and $3.0 \mathrm{~g} / \mathrm{l}$ Merck quality ethylalcohol concentrations. They were analysed with a Perkin-Elmer F42 gas chromatograph, which was equipped with an automatic headspace sampler. The sample sequences contained control samples introduced in a random manner in order to produce the autocorrelation-type error component. Flushing was used to remove the remaining part of the sample from the sampling capillary and LAB pipettes were used for sampling. The internal standard method was used for evaluation and a solution of $0.5 \mathrm{~g} / \mathrm{l} \mathrm{l-propanol} \mathrm{was} \mathrm{added} \mathrm{to} \mathrm{each} \mathrm{sample} \mathrm{for} \mathrm{this}$ purpose. To avoid systematic error due to sampling, the internal standard was added to the sample with the same pipette and pipette-tip in all cases.

The sample sequences were repeatedly measured under different conditions. The parameters varied were sampling with the same or different pipettes and tips, and the duration of the flush.

Measurements were performed under the following conditions:

(1) Sequence: sampling with the same pipette (the first source of autocorrelation-type error) and with the same tip (the second source of autocorrelation-type error), $0 \mathrm{~s}$ flush (no flush) (the third source of autocorrelation type error);

(2) Sequence: sampling with the same pipette but three different tips, $0 \mathrm{~s}$ flush;

(3) Sequence: sampling with the same pipette and tip, $15 \mathrm{~s}$ flush (a weak source of autocorrelation-type error);

(4) Sequence: sampling with three different pipettes and three different tips, $15 \mathrm{~s}$ flush.

The evaluation of the chromatograms was performed by two separate methods: based on the ratios of peak areas or that of peak heights of ethylalcohol and the internal standard.

The model of the measurement error sequence: the autocorrelation-type component

The sequence of the measured values can be denoted by:

$$
\xi_{t}, \quad t=1,2, \ldots N
$$

and the true values of the sample sequence by

$$
X_{t}, \quad t=1,2, \ldots, N
$$

for an automatically analysed measurement-sequence.

Their difference-sequence:

$$
x_{t}=\xi_{t}-X_{t}, \quad t=1,2, \ldots N
$$

can be called the 'measurement error sequence'. It is usually divided into a number of components (drift, random measurement error etc.), which have different physical and mathematical properties. 
Several papers have been published recently on modelling measurement error processes in continuous-flow systems and in discrete samples. Wijtoliet [2] has thoroughly investigated measurement errors in gas chromatography, and several attempts have also been made to construct a generally valid measurement error process model [3 and 4].

The measured value sequence, as well as the measurement error sequence, can be modelled using discrete time stochastic processes (stochastic sequences). It can be shown that under relatively weak assumptions (assuming no discontinuity in the trajectories of the investigated processes with probability 1 and the Markov-property), the continuous time measurement error and measured value processes (in the case of continuously-flow samples) can be described by Ito-processes [5]. The discrete time model, i.e. the measurement error and measured value sequences can be derived from this model by applying equidistant sampling [6]. This discrete time model takes the form:

$$
\xi_{t}=m\left(\xi_{t-1}, t\right)+\delta\left(\xi_{t}, t\right)^{*} \varepsilon_{t}
$$

where

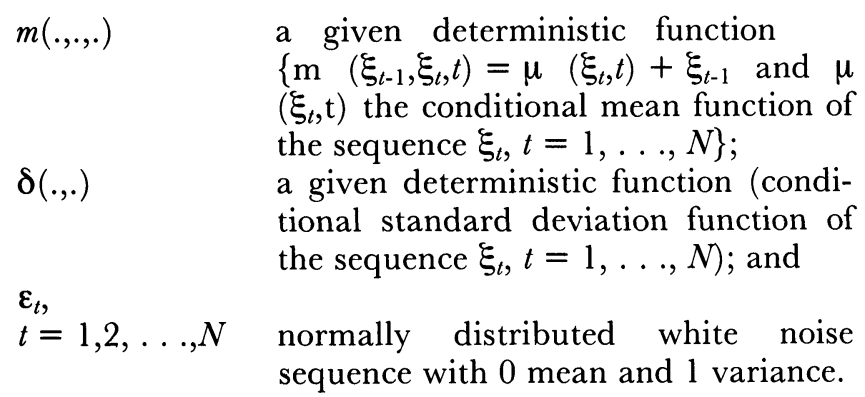

In order to get simpler formulae functions $m(., .,$.$) and$ $\sigma(.,$.$) in equation (4) are expanded in powers of t$, and the terms of first order (for $\sigma$ ) and that of second order for $m$ ) are neglected. Thus the following equation is obtained from equation (4):

$$
\xi_{t}=A\left(\xi_{t-1}, \xi_{t}\right)+\mathrm{M}^{*} t+\mathrm{S}^{*} \varepsilon_{t}+X_{t}
$$

where

$A(.,$.$) \quad is a given deterministic function \{$ depending on $m(., .,)$.$\} ; and$

$\mathrm{M}$ and $\mathrm{S}$ are constants.

There are three different terms on the right-hand side of equation (5) with different mathematical properties. The second and third terms do not depend on the previous samples, nor on the previous time; the first term depends on both the $t$ th and on the previous sample. Thus the first term can be regarded as a general model of the autocorrelation-type error components. The second term is a fully deterministic linear function of time, so it can express a very simple linear drift. At the same time the third term is a fully stochastic, 0 mean white noise (with independent elements) sequence, which can be regarded as a random measurement error component.

The most simple linearized model of the measured value sequence can be obtained from equation (5) assuming the most simple linear form of the autocorrelation-type error component:

$$
A\left(\xi_{t-1}, \xi_{t}\right)=a_{0}+a^{*}\left(\xi_{t}-\xi_{t-1}\right) .
$$

Substituting equation (6) into equation (5) gives:

$$
\xi_{t}=a_{0}+a^{*}\left(\xi_{t}-\xi_{t-1}\right)+\mathrm{M}^{*} t+\mathrm{S}^{*} \varepsilon_{t}+\mathrm{x}_{t} .
$$

Applying the definition of the measurement error sequence results in the following equation:

$$
x_{\mathrm{t}}=a_{0}+\mathrm{M}^{*} t+a\left(\xi_{t}-\xi_{t-1}\right)+\mathrm{S}^{*} \varepsilon_{t} .
$$

Note that in almost all practical cases, the standard deviation of the random measurement error component (S) and the mean of the measurement error $\left(a_{0}+\mathrm{M}^{*} t\right)$ depend on the true value $X_{i}$ :

$$
\mathrm{S}=\mathrm{S}\left(X_{t}\right) \text { and } a_{0}+\mathrm{M}^{*} t=A_{0}\left(X_{t}\right) .
$$

\section{Detection of the autocorrelation-type error com- ponent}

Detection requires testing the hypothesis on the presence of the autocorrelation-type measurement error component based on the mathematical model (equation (5)). The absence of the autocorrelation-type component is mathematically equivalent with the equality $a=0$.

It can also be seen from equation (8) that the conditional probability distribution of the measurement error $x_{t}$, conditioned on the difference $\left(\xi_{t}-\xi_{t-1}\right)$, is Gaussian with a mean $\left(\mathbf{M}^{*} t+a_{0}\right)$ and with a variance $\mathbf{S}^{2}$. So the following simple hypothesis can be investigated in practical cases instead of $a=0$.

Hypothesis 1: the conditional mean and the conditional variance of the measurement error sequence conditioned on the difference $\left(\xi_{t}-\xi_{t-1}\right)$ are independent of the condition.

In order to test this hypothesis, the domain of the possible values in the difference $\left(\xi_{t}-\xi_{t-1}\right)$ must be divided by the points:

$$
\xi_{1}<\xi_{2}<\ldots<\xi_{K} .
$$

With the help of these points, hypothesis 1, can be approximated by a set of other hypotheses as follows:

Hypothesis 2:

$$
\begin{gathered}
E\left[x_{t} \mid \rho_{i} \leqslant\left(\xi_{t}-\xi_{t-1}\right)<\rho_{i+1}\right]=E\left[x_{t}\right] \\
E\left[\mathrm{O} x_{t}^{2} \mid \rho_{i} \leqslant\left(\xi_{t}-\xi_{t-1}\right)<\rho_{i+1}\right]=E\left[x_{t}^{2}\right] \\
i=1,2, \ldots, K-1
\end{gathered}
$$

It is important to note that the set of hypothesis 2 is only an approximation of hypothesis 1 , but it has a great practical 'advantage in this form'. As it can be seen from equation (11), the hypothesis can be easily tested with known statistical tests (F-test and t-test) and the necessary values in the condition can be easily computed from the measured value sequence, $\xi_{\iota}$, itself. The only problem from the computational viewpoint is how the values of the measurement error $x_{t}$ can be computed.

For this purpose, the control samples can be used because their true values, $x$, are assumed to be known. In this case it is assumed that the error of the control liquid sample preparation is negligible compared to the effects of the other measurement error components. 
In the case of automatic analysis of large analytical sample sequences, control samples occur rarely in the sample sequences compared to the unknown samples. Thus the points in equation (10) must be chosen very carefully in order to have enough sample for testing each hypothesis in set 2 (equation (11)) for a good approximation of hypothesis 1 .

In order to verify the numerical method, sample sequences containing only control samples were used in the authors experiments. This results in an increased number of suitable samples in the measurement error and in an obvious choice of the points in equation (10). The control samples with $0.5,1.0$, and $3.0 \mathrm{~g} / 1$ ethylalcohol concentrations can produce differences $\left(\xi_{t}-\xi_{t-1}\right)$ only in the region of the following values:

$$
0.0,0.5,-0.5,2.0,-2.0,2.5,-2.5
$$

thus the values can be chosen as:

$$
\begin{aligned}
& \rho_{1}=-3.0 ; \quad \rho_{2}=-2.25 ; \quad \rho_{3}=-1.5 ; \quad \rho_{4}=-0.25 ; \\
& \rho_{5}=0.25 ; \quad \rho_{6}=1.5 ; \quad \rho_{7}=2.25 ; \quad \rho_{8}=3.0 .
\end{aligned}
$$

\section{The proposed method and its application to control sample sequences}

In order to show how tests for the set of hypotheses given in equation (11) can be performed easily, the computed quantities needed for the numerical method have been collected and arranged in tables $1-4$, according to the four sample sequences described previously. The quantities in tables 1-4 have been computed from the measured values as follows.
A row in a table belongs to a given control sample concentration and to a given evaluation method (for example $0.5 \mathrm{~g} / \mathrm{l}$ ethylalcohol concentration and ethylalcohol/internal standard peak area ratio evaluation).

The test of the hypothesis 2 set for each row is done in three steps:

(1) The number, the empirical mean and the empirical variance of the samples with the given concentration is computed and placed in the fourth column. After this, these samples are divided into three groups according to the previous sample concentration. The above characteristics (number, empirical mean and empirical variance) of each group is computed and put into columns one to three respectively.

(2) F-tests [7] can be used for testing hypothesis 2 (equation (11) for the variances. It is sufficient, however, to perform the test for the ratio of the columns with maximal and minimal variances. The computed F-value, together with the result of the hypothesis test, is put to the fifth column. The result of a test is positive ('+' sign) if the hypothesis has been proved true on the given significance level.

(3) If the result of the F-test is positive, the two sample t-tests can be applied to discover whether hypothesis 2 (equation [11]) holds for the mean values. In this case, it is also sufficient to perform the test for the columns with minimal and maximal means, applying the empirical variance of the fourth column as a common variance. The computed t-value and the result of the

\begin{tabular}{|c|c|c|c|c|c|c|c|c|c|}
\hline $\begin{array}{c}\text { Sample } \\
\text { concentration } \\
(\mathrm{g} / \mathrm{l})\end{array}$ & & $X_{t-1}=0.5$ & $X_{t-1}=1 \cdot 0$ & $X_{t-1}=3 \cdot 0$ & $\begin{array}{l}\text { Uncondi- } \\
\text { tioned }\end{array}$ & $F$ & $i / n$ & $t$ & $i / n$ \\
\hline \multicolumn{10}{|c|}{ Peak height ratio of ethylalcohol/1-propanol } \\
\hline \multirow[t]{3}{*}{$0 \cdot 5$} & $\bar{x}$ & 0.5166 & 0.5219 & 0.5190 & 0.5194 & $11 \cdot 03$ & & & \\
\hline & $s_{x}^{2}$ & $1 \cdot 1184 \mathrm{E}-4$ & $1 \cdot 2330 \mathrm{E}-3$ & $4 \cdot 0815 \mathrm{E}-4$ & $5 \cdot 9980 \mathrm{E}-4$ & & & & \\
\hline & $n$ & 12 & 15 & 14 & 41 & & - & & \\
\hline \multirow[t]{3}{*}{$1 \cdot 0$} & $\bar{x}$ & $1 \cdot 0157$ & $1 \cdot 0185$ & $1 \cdot 0008$ & $1 \cdot 0137$ & $1 \cdot 87$ & & 0.7074 & \\
\hline & $s_{x}^{2}$ & $1.9843 \mathrm{E}-3$ & $2 \cdot 9988 \mathrm{E}-3$ & $3 \cdot 5366 \mathrm{E}-3$ & $2 \cdot 5432 \mathrm{E}-3$ & & & & \\
\hline & $n$ & 18 & 14 & 8 & 40 & & + & & + \\
\hline \multirow[t]{3}{*}{$3 \cdot 0$} & $\bar{x}$ & $3 \cdot 0931$ & $3 \cdot 1218$ & $3 \cdot 1742$ & $3 \cdot 1236$ & 1.72 & & 0.6772 & \\
\hline & $s_{x}^{2}$ & $6 \cdot 8099 \mathrm{E}-2$ & $3.9616 \mathrm{E}-2$ & $5 \cdot 0129 \mathrm{E}-2$ & $5 \cdot 0220 \mathrm{E}-2$ & & & & \\
\hline & $n$ & 11 & 11 & 7 & 29 & & + & & + \\
\hline \multicolumn{10}{|c|}{ Peak area ratio of ethylalcohol/1-propanol } \\
\hline \multirow[t]{3}{*}{$0 \cdot 5$} & $\bar{x}$ & 0.5764 & 0.5737 & 0.5791 & 0.5763 & $1 \cdot 31$ & & $2 \cdot 302$ & \\
\hline & $s_{x}^{2}$ & $4 \cdot 4996 \mathrm{E}-5$ & $4 \cdot 4863 \mathrm{E}-5$ & $3 \cdot 4438 \mathrm{E}-5$ & $4 \cdot 4660 \mathrm{E}-5$ & & & & \\
\hline & $n$ & 12 & 15 & 14 & 41 & & + & & \\
\hline \multirow[t]{3}{*}{$1 \cdot 0$} & $\bar{x}$ & $1 \cdot 1369$ & $1 \cdot 1340$ & $1 \cdot 1405$ & $1 \cdot 1366$ & $5 \cdot 86$ & & & \\
\hline & $s_{x}^{2}$ & $9 \cdot 7885 \mathrm{E}-4$ & $1 \cdot 6715 \mathrm{E}-4$ & $9 \cdot 0286 \mathrm{E}-4$ & $6 \cdot 4960 \mathrm{E}-4$ & & & & \\
\hline & $n$ & 18 & 14 & 8 & 40 & & - & & \\
\hline \multirow[t]{3}{*}{$3 \cdot 0$} & $\bar{x}$ & $3 \cdot 4202$ & 3.4089 & 3.3993 & 3.4109 & $4 \cdot 82$ & & $0 \cdot 8595$ & \\
\hline & $s_{x}^{2}$ & $4 \cdot 2738 \mathrm{E}-3$ & $8 \cdot 8749 \mathrm{E}-4$ & $2 \cdot 2308 \mathrm{E}-3$ & $2 \cdot 3893 \mathrm{E}-3$ & & & & \\
\hline & $n$ & 11 & 11 & 7 & 29 & & + & & + \\
\hline
\end{tabular}
test can be found in the last column.

Table 1. Data from measurement sequence 1. 
Table 2. Data from measurement sequence 2.

\begin{tabular}{|c|c|c|c|c|c|c|c|c|c|}
\hline $\begin{array}{c}\text { Sample } \\
\text { concentration } \\
(\mathrm{g} / 1)\end{array}$ & & $X_{t-1}=0.5$ & $X_{t-1}=1 \cdot 0$ & $X_{t-1}=3 \cdot 0$ & Unconditioned & $F$ & $i / n$ & $t$ & $i / n$ \\
\hline 0.5 & $\begin{array}{c}\bar{x} \\
s_{x}^{2} \\
n\end{array}$ & $\begin{array}{r}\mathrm{Pe} \\
0 \cdot 5069 \\
3 \cdot 6267 \mathrm{E}-4 \\
14\end{array}$ & $\begin{array}{c}\text { eight ratio of } \\
0.5161 \\
1.9730 \mathrm{E}-3 \\
17\end{array}$ & $\begin{array}{c}\text { alcohol/1-pr } \\
0 \cdot 5054 \\
5 \cdot 1038 \mathrm{E}-4 \\
19\end{array}$ & $\begin{array}{c}0.5095 \\
9 \cdot 5145 \mathrm{E}-4 \\
50\end{array}$ & $5 \cdot 44$ & - & & \\
\hline $1 \cdot 0$ & $\begin{array}{c}\bar{x} \\
s_{x}^{2} \\
n\end{array}$ & $\begin{array}{c}1 \cdot 036 \\
1 \cdot 2067 \mathrm{E}-3 \\
19\end{array}$ & $\begin{array}{c}1 \cdot 0290 \\
3 \cdot 0079 \mathrm{E}-4 \\
18\end{array}$ & $\begin{array}{c}1 \cdot 0143 \\
5 \cdot 1722 \mathrm{E}-3 \\
13\end{array}$ & $\begin{array}{c}1 \cdot 0279 \\
1 \cdot 8892 \mathrm{E}-3 \\
50\end{array}$ & $17 \cdot 18$ & - & & \\
\hline $3 \cdot 0$ & $\begin{array}{c}\bar{x} \\
s_{x}^{2} \\
n\end{array}$ & $\begin{array}{c}3 \cdot 0605 \\
2 \cdot 1116 \mathrm{E}-2 \\
18\end{array}$ & $\begin{array}{c}3 \cdot 0879 \\
1 \cdot 2772 \mathrm{E}-4 \\
15\end{array}$ & $\begin{array}{c}3 \cdot 0443 \\
1 \cdot 2759 \mathrm{E}-2 \\
17\end{array}$ & $\begin{array}{c}3 \cdot 0632 \\
1 \cdot 1839 \mathrm{E}-2 \\
50\end{array}$ & $165 \cdot 33$ & - & & \\
\hline $0 \cdot 5$ & $\begin{array}{c}\bar{x} \\
s_{x}^{2} \\
n\end{array}$ & $\begin{array}{c}0.5766 \\
5 \cdot 2546 \mathrm{E}-4 \\
14\end{array}$ & $\begin{array}{c}\text { area ratio of e } \\
0.5972 \\
3 \cdot 5678 \mathrm{E}-3 \\
17\end{array}$ & $\begin{array}{c}\text { lcohol/1-pro } \\
0 \cdot 5733 \\
2 \cdot 0410 \mathrm{E}-4 \\
19\end{array}$ & $\begin{array}{c}\text { nol } \\
0.5823 \\
1.4971 \mathrm{E}-3 \\
50\end{array}$ & $17 \cdot 48$ & - & & \\
\hline $1 \cdot 0$ & $\begin{array}{c}\bar{x} \\
s_{x}^{2} \\
n\end{array}$ & $\begin{array}{c}1 \cdot 1738 \\
1 \cdot 9391 \mathrm{E}-3 \\
19\end{array}$ & $\begin{array}{c}1 \cdot 1541 \\
5 \cdot 0186 \mathrm{E}-4 \\
18\end{array}$ & $\begin{array}{c}1 \cdot 1814 \\
2 \cdot 1103 \mathrm{E}-3 \\
13\end{array}$ & $\begin{array}{c}1 \cdot 1687 \\
1 \cdot 5333 \mathrm{E}-3 \\
50\end{array}$ & $4 \cdot 20$ & - & & \\
\hline $3 \cdot 0$ & $\begin{array}{c}\bar{x} \\
s_{x}^{2} \\
n\end{array}$ & $\begin{array}{c}3 \cdot 4997 \\
1 \cdot 3274 \mathrm{E}-2 \\
18\end{array}$ & $\begin{array}{c}3 \cdot 4887 \\
8 \cdot 3348 \mathrm{E}-3 \\
15\end{array}$ & $\begin{array}{c}3 \cdot 4507 \\
8 \cdot 5298 \mathrm{E}-3 \\
17\end{array}$ & $\begin{array}{c}3 \cdot 4797 \\
1 \cdot 0238 \mathrm{E}-2 \\
50\end{array}$ & $1 \cdot 65$ & + & $1 \cdot 383$ & \\
\hline
\end{tabular}

Table 3. Data from measurement sequence 3.

\begin{tabular}{|c|c|c|c|c|c|c|c|c|c|}
\hline $\begin{array}{c}\text { Sample } \\
\text { concentration } \\
(\mathrm{g} / 1)\end{array}$ & & $X_{t-1}=0.5$ & $X_{t-1}=1 \cdot 0$ & $X_{t-1}=3.0$ & Unconditioned & $F$ & $i / n$ & $t$ & $i / n$ \\
\hline 0.5 & $\begin{array}{c}\bar{x} \\
s_{x}^{2} \\
n\end{array}$ & $\begin{array}{r}\mathrm{Pe} \\
0.5194 \\
1 \cdot 6558 \mathrm{E}-4 \\
14\end{array}$ & $\begin{array}{c}\text { eight ratio of } \\
0 \cdot 5062 \\
1 \cdot 3228 \mathrm{E}-3 \\
17\end{array}$ & $\begin{array}{c}\text { lcohol/1-pr } \\
0.5194 \\
1.0286 \mathrm{E}-5 \\
19\end{array}$ & $\begin{array}{c}\text { anol } \\
\begin{array}{c}0 \cdot 5149 \\
5 \cdot 1929 \mathrm{E}-4 \\
50\end{array}\end{array}$ & $128 \cdot 57$ & - & & \\
\hline $1 \cdot 0$ & $\begin{array}{c}\bar{x} \\
s_{x}^{2} \\
n\end{array}$ & $\begin{array}{c}1 \cdot 0540 \\
1 \cdot 4988 \mathrm{E}-3 \\
19\end{array}$ & $\begin{array}{c}1 \cdot 0552 \\
6 \cdot 9119 \mathrm{E}-4 \\
18\end{array}$ & $\begin{array}{c}1 \cdot 0447 \\
5 \cdot 3982 \mathrm{E}-5 \\
13\end{array}$ & $\begin{array}{c}1 \cdot 0520 \\
8 \cdot 2303 \mathrm{E}-4 \\
50\end{array}$ & $27 \cdot 76$ & - & & \\
\hline $3 \cdot 0$ & $\begin{array}{c}\bar{x} \\
s_{x}^{2} \\
n\end{array}$ & $\begin{array}{c}3 \cdot 0809 \\
4 \cdot 1502 \mathrm{E}-4 \\
18\end{array}$ & $\begin{array}{c}3 \cdot 1046 \\
6 \cdot 0030 \mathrm{E}-3 \\
15\end{array}$ & $\begin{array}{c}3 \cdot 0820 \\
1 \cdot 4024 \mathrm{E}-3 \\
17\end{array}$ & $\begin{array}{c}3 \cdot 0884 \\
2 \cdot 4348 \mathrm{E}-3 \\
50\end{array}$ & $14 \cdot 46$ & - & & \\
\hline $0 \cdot 5$ & $\begin{array}{c}\bar{x} \\
s_{x}^{2} \\
n\end{array}$ & $\begin{array}{c}0.6247 \\
4 \cdot 8197 \mathrm{E}-4 \\
14\end{array}$ & $\begin{array}{c}\text { area ratio of e } \\
0 \cdot 6234 \\
5 \cdot 7022 \mathrm{E}-4 \\
17\end{array}$ & $\begin{array}{c}\text { lcohol/1-pro } \\
0 \cdot 6218 \\
4 \cdot 5042 \mathrm{E}-4 \\
19\end{array}$ & $\begin{array}{c}\text { nol } \\
\begin{array}{c}0.6231 \\
4 \cdot 8094 \mathrm{E}-4 \\
50\end{array}\end{array}$ & $1 \cdot 26$ & + & $0 \cdot 3824$ & + \\
\hline $1 \cdot 0$ & $\begin{array}{c}\bar{x} \\
s_{x}^{2} \\
n\end{array}$ & $\begin{array}{c}1 \cdot 2551 \\
1 \cdot 4420 \mathrm{E}-3 \\
19\end{array}$ & $\begin{array}{c}1 \cdot 2534 \\
1 \cdot 4008 \mathrm{E}-3 \\
18\end{array}$ & $\begin{array}{c}1 \cdot 2856 \\
1.9363 \mathrm{E}-3 \\
13\end{array}$ & $\begin{array}{c}1 \cdot 2624 \\
1 \cdot 6830 \mathrm{E}-3 \\
50\end{array}$ & $1 \cdot 38$ & + & $2 \cdot 196$ & \\
\hline $3 \cdot 0$ & $\begin{array}{c}\bar{x} \\
s_{x}^{2} \\
n\end{array}$ & $\begin{array}{c}3 \cdot 7425 \\
1 \cdot 2728 \mathrm{E}-2 \\
18\end{array}$ & $\begin{array}{c}3 \cdot 7303 \\
1 \cdot 1374 \mathrm{E}-2 \\
15\end{array}$ & $\begin{array}{c}3 \cdot 7677 \\
1 \cdot 5252 \mathrm{E}-2 \\
17\end{array}$ & $\begin{array}{c}3 \cdot 7474 \\
1 \cdot 2883 \mathrm{E}-2 \\
50\end{array}$ & $1 \cdot 34$ & + & 0.9106 & + \\
\hline
\end{tabular}

By devoting separate rows to each control sample's concentration, the dependence of the measurement error mean and variance (equation (9)) on the true value of the samples is taken into account.

As the result of the above procedure, there is no autocorrelation-type measurement error component under the given measurement circumstances and evaluation method if all the results of the corresponding F- and $\mathrm{t}$-tests are positive on the given significance level.

It is important to note that the security of the autocorrelation-type measurement error component testing is influenced by the value of the $\mathrm{S}^{2}\left(X_{l}\right)$ measurement error 
Table 4. Data from measurement sequence 4.

\begin{tabular}{|c|c|c|c|c|c|c|c|c|c|}
\hline $\begin{array}{c}\text { Sample } \\
\text { concentration } \\
\mathrm{g} / 1\end{array}$ & & $X_{t-1}=0.5$ & $X_{t-1}=1 \cdot 0$ & $X_{t-1}=3 \cdot 0$ & Unconditioned & $F$ & $i / n$ & $t$ & $i / n$ \\
\hline \multicolumn{10}{|c|}{ Peak height ratio of ethylalcohol/1-propanol } \\
\hline \multirow[t]{3}{*}{$0 \cdot 5$} & $\bar{x}$ & $0 \cdot 5191$ & 0.5177 & 0.5204 & $0 \cdot 5185$ & $3 \cdot 23$ & & & \\
\hline & $s_{x}^{2}$ & $9 \cdot 9173 \mathrm{E}-6$ & $1 \cdot 3014 \mathrm{E}-5$ & $4 \cdot 0266 \mathrm{E}-6$ & $1 \cdot 0522 \mathrm{E}-5$ & & & & \\
\hline & $n$ & 14 & 17 & 19 & 50 & & - & & \\
\hline \multirow[t]{3}{*}{$1 \cdot 0$} & $\bar{x}$ & $1 \cdot 0154$ & $1 \cdot 0171$ & $1 \cdot 0221$ & $1 \cdot 0177$ & 3.92 & & & \\
\hline & $s_{x}^{2}$ & $1 \cdot 9756 \mathrm{E}-5$ & $2 \cdot 9915 \mathrm{E}-5$ & $7 \cdot 7638 \mathrm{E}-5$ & $4 \cdot 3323 \mathrm{E}-5$ & & & & \\
\hline & $n$ & 19 & 18 & 12 & 49 & & - & & \\
\hline \multirow[t]{3}{*}{$3 \cdot 0$} & $\bar{x}$ & $3 \cdot 0245$ & $3 \cdot 0287$ & $3 \cdot 0243$ & 3.0256 & $1 \cdot 07$ & & $0 \cdot 9329$ & \\
\hline & $s_{x}^{2}$ & $1 \cdot 8883 \mathrm{E}-4$ & $1 \cdot 7665 \mathrm{E}-4$ & $1 \cdot 8120 \mathrm{E}-4$ & $1 \cdot 8385 \mathrm{E}-4$ & & & & \\
\hline & $n$ & 18 & 14 & 19 & 51 & & + & & + \\
\hline \multicolumn{10}{|c|}{ Peak area ratio of ethylalcohol/1-propanol } \\
\hline \multirow[t]{3}{*}{$0 \cdot 5$} & $\bar{x}$ & U.6087 & $0 \cdot 6167$ & 0.6158 & $0 \cdot 6141$ & $1 \cdot 17$ & & 1.073 & \\
\hline & $s_{x}^{2}$ & $3 \cdot 8976 \mathrm{E}-4$ & $4 \cdot 5753 \mathrm{E}-4$ & $4 \cdot 0974 \mathrm{E}-4$ & $4 \cdot 1535 \mathrm{E}-4$ & & & & \\
\hline & $n$ & 14 & 17 & 19 & 50 & & + & & + \\
\hline \multirow[t]{3}{*}{$1 \cdot 0$} & $\bar{x}$ & $1 \cdot 2155$ & $1 \cdot 2097$ & $1 \cdot 2285$ & $1 \cdot 2166$ & $1 \cdot 69$ & & $1 \cdot 071$ & \\
\hline & $s_{x}^{2}$ & $2 \cdot 0396 \mathrm{E}-3$ & $2 \cdot 6465 \mathrm{E}-3$ & $1.5623 \mathrm{E}-3$ & $2 \cdot 1147 \mathrm{E}-3$ & & & & \\
\hline & $n$ & 19 & 18 & 12 & 49 & & + & & + \\
\hline \multirow[t]{3}{*}{$3 \cdot 0$} & $\bar{x}$ & $3 \cdot 6190$ & 3.5948 & $3 \cdot 6492$ & $3 \cdot 6236$ & $1 \cdot 89$ & & $1 \cdot 152$ & \\
\hline & $s_{x}^{2}$ & $1 \cdot 0940 \mathrm{E}-2$ & $1 \cdot 4232 \mathrm{E}-2$ & $2 \cdot 0689 \mathrm{E}-2$ & $1 \cdot 5352 \mathrm{E}-2$ & & & & \\
\hline & $\hat{n}$ & 18 & 14 & 19 & 51 & & + & & + \\
\hline
\end{tabular}

variance. The smaller that variance, the smaller the effect that can be detected with a given sample size and with a given significance level.

\section{Conclusions}

By applying this numerical and graphical (LAG-1) method for detecting autocorrelation-type measurement errors, it is evident that the results are the same for both methods.

In the case of the peak height ratio evaluation method, there were no such measurement circumstances when no autocorrelation-type measurement error component was present.

When applying the peak area ratio evaluation method, sequences three and four have been shown to have no autocorrelation-type measurement error component according to the numerical (see tables 3 and 4) and the graphical methods. This indicates that the circumstances of the flush have much more influence on the measurement error than do the other measurement circumstances (pipettes).

From the data of the numerical method (tables 1 and 2), it can also be seen that the empirical variances of the ratio of the peak height is much smaller than that of the peak areas. This fact is in good agreement with previous investigations [8]. As a consequence, the use of the peak height ratio allows the detection of smaller autocorrelation-type measurement error components than would be possible in the case of the ratio of the peak areas. At the same time, it can also be found that the empirical variance of the samples is much more influenced by the difference of the current and previous measured value, than the empirical mean of them, i.e. the autocorrelationtype measurement error component appears much more sensistive in the empirical variance.

The numerical method proposed here can be used for automatic validation and quality control of analytical measurements.

\section{References}

1. Filliben, J. J., In Validation of Measurement Process, Ed. De Voe (ACS Symposium Series 63, American Chemical Society, Washington, D.C., 1977).

2. Wijtoliet, J. J. M., Ultimate Retention Time Accuracy in Computer Assisted Chromatography (Thesis, Eindhoven, 1972).

3. Currie, L. A., In Treatise on Analytical Chemistry Eds I. M. Kolthoff and P. J. Elving (Part 1, Vol. 1, 2nd edn, John Wiley and Sons, New York, 1978), 95.

4. Hangos, K. M., Chemical Engineering Science, 39 (1984), 1233.

5. Jazwinski, A. H., Stochastic processes and Fitering Theory (Academic Press, New York and London, 1970).

6. Hangos, K. M., 9th IFAC World Congress $\mathbf{X}$ (Budapest, 1984), 39 .

7. RAO, C. R., Linear Statistical Inference and Its Applications (2nd edn, John Wiley and Sons, New York, London, Sydney and Toronto, 1973).

8. Leisztner, L., Kuzmin, N. M. and Barna, P., Zh Anal. Khim., 38 (1983), 2247. 


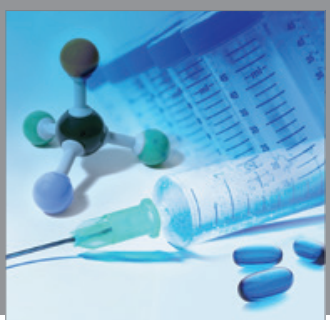

International Journal of

Medicinal Chemistry

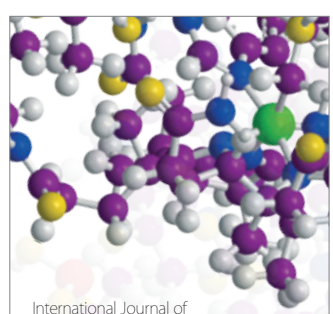

Carbohydrate Chemistry

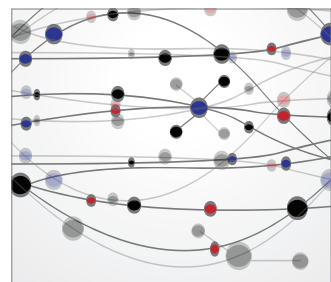

The Scientific World Journal
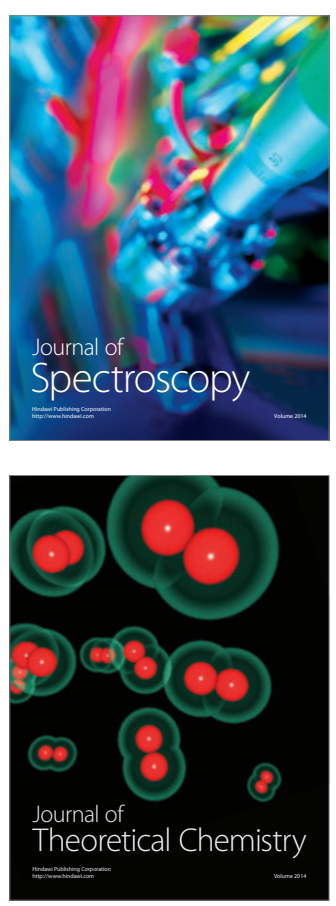
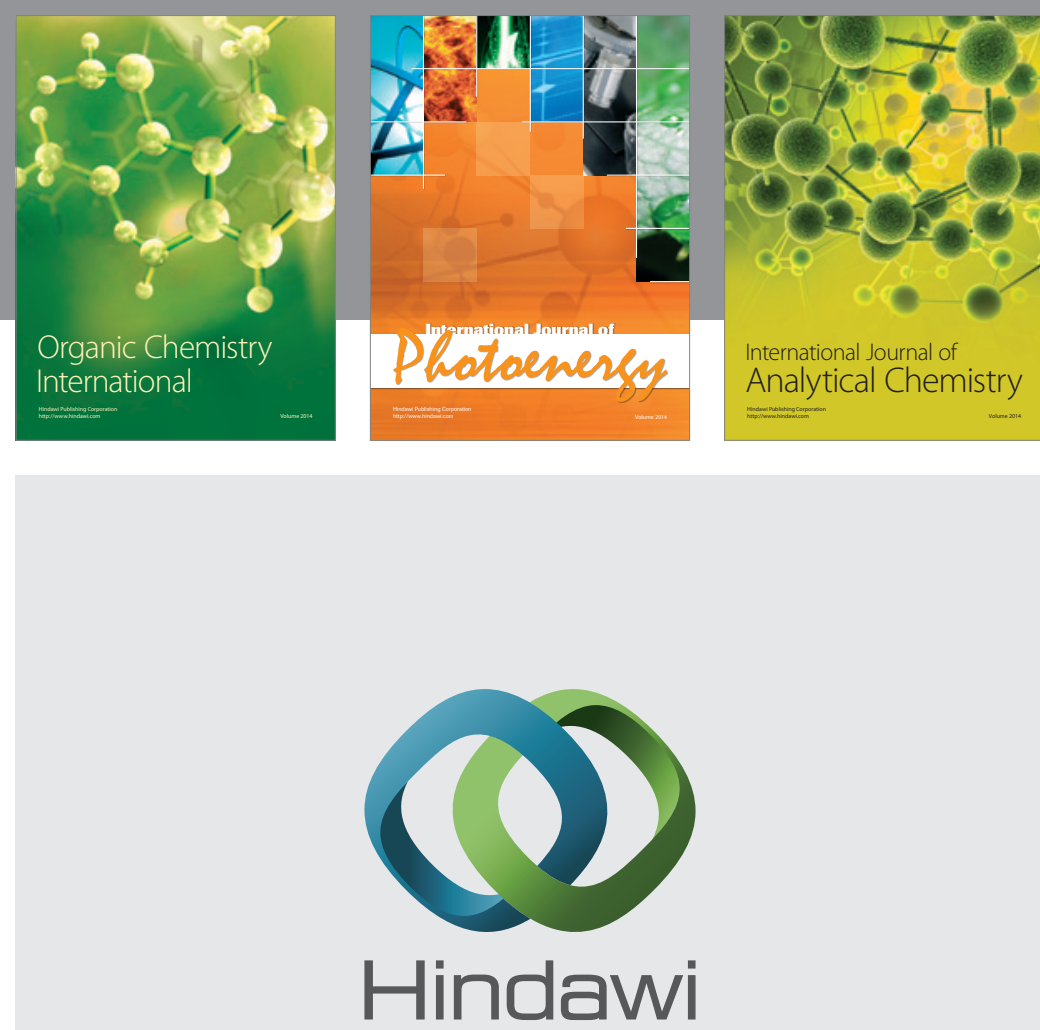

Submit your manuscripts at

http://www.hindawi.com
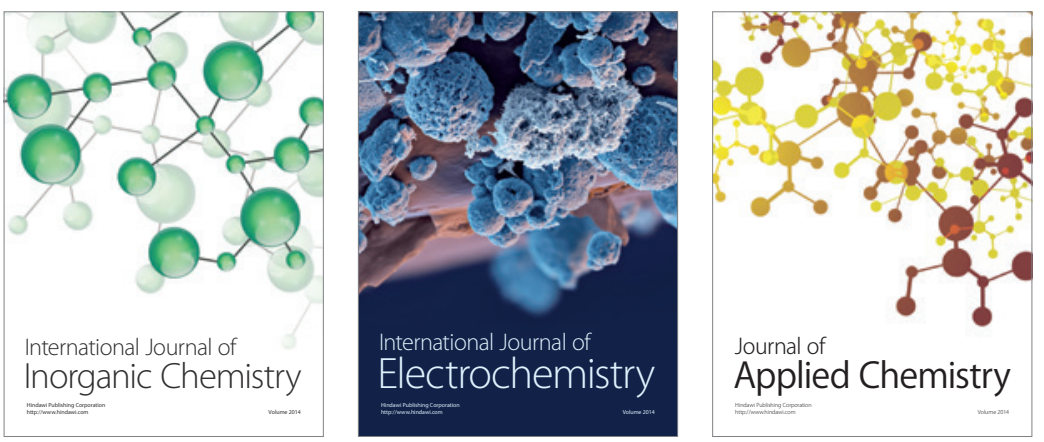

Journal of

Applied Chemistry
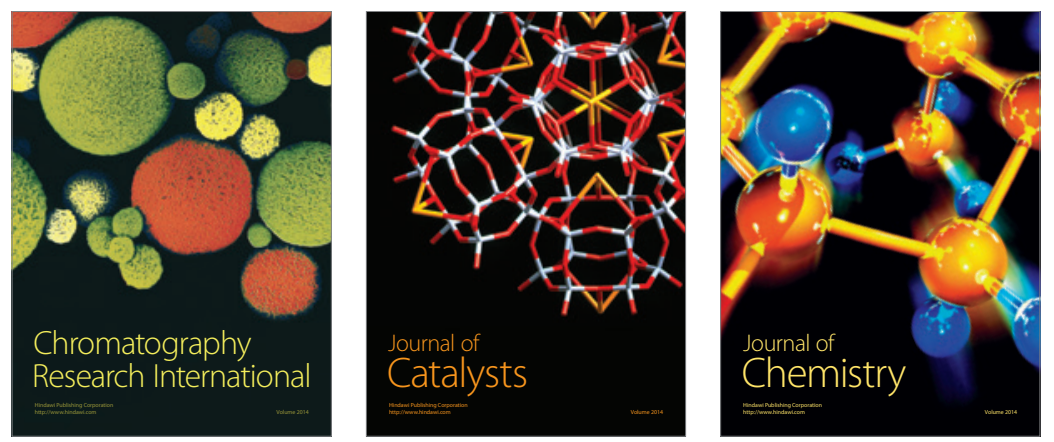
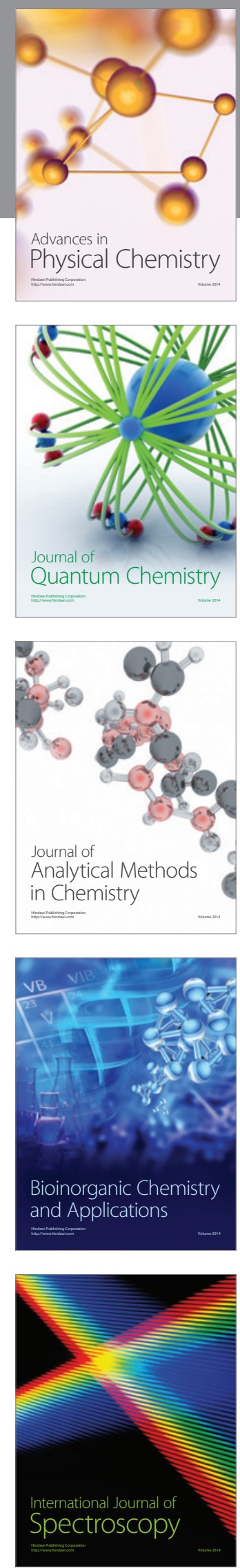